\title{
Modeling of Printed Spiral Inductors for Remote Powering of Implantable Biosensors
}

\author{
Jacopo Olivo, Sandro Carrara, and Giovanni De Micheli \\ Integrated Systems Laboratory \\ École Polytechnique Fédérale de Lausanne - EPFL, Lausanne, Switzerland \\ email: jacopo.olivo@epfl.ch
}

\begin{abstract}
Fully implantable biosensors require small size to be minimally invasive. To avoid embedded batteries, power can be supplied by means of printed spiral inductors located on the skin, close to the implanted devices. Reliable models are required to optimize the design of such inductors. In this paper, a RLC model to describe the electrical properties of printed spiral inductors is proposed. The model is based on the geometrical and physical characteristics of the inductors. The accuracy of the model is finally compared with the experimental measurements.
\end{abstract}

\section{INTRODUCTION}

Remote and continuous monitoring of relevant human metabolites, such as glucose and lactate, is a prerequisite to enable personalized therapies. This kind of treatments are calibrated on the response of the patient and require a constant "telemetry" of his/her health status. Implantable biosensors represent a possible solution to enable a minimally-invasive, constant monitoring of the patient.

In order to realize such scenarios, several issues must be addressed. Biosensors implanted in the subcutaneous tissues must be biocompatible, with low thermal dissipation, low invasivity and with large power autonomy. This last requirement can be satisfied by using energy harvesting methods. These techniques exploit natural and/or artificial power sources surrounding the person to help, and in certain cases substitute implanted batteries.

Among the different techniques presented in the literature, we focus on inductive remote powering. By exploiting the magnetic coupling between two inductors, named transmitter and receiver, an alternate current is induced in the receiving inductor by means of an alternate current flowing in the transmitting inductor [1]. Thus, with the receiving inductor implanted in the subcutaneous tissues and the transmitting inductor located just over the implantation area, power can be wirelessly transmitted to the biosensor through the body tissues [2].

In a previous work [3], we have studied the use of receiving inductors having form factor comparable with a needle. In that work, we have shown that the optimal operating frequency is strongly affected by the geometries of the inductors, while it is not considerably affected by the different kinds of subcutaneous tissues between the inductors. By using a single-turn implanted inductor with an area of $9 \mathrm{~mm}^{2}$ and a single-turn external inductor with an area of $120 \mathrm{~mm}^{2}$, simulations have shown an optimal operating frequency around $400 \mathrm{MHz}$, two

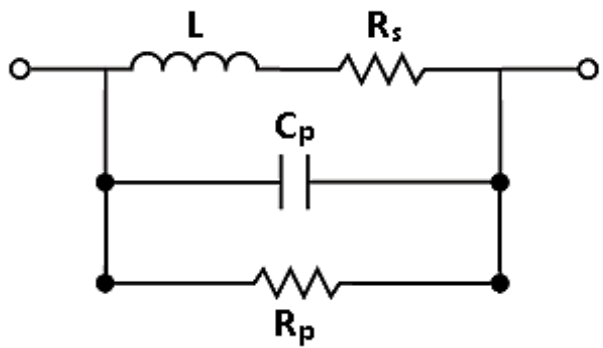

Fig. 1. Lumped RLC model of a printed spiral inductor. Second order effects are not considered.

orders of magnitude higher than the frequencies commonly used for inductive powering. However, by increasing the size of the external inductor and the number of turns, the optimal working frequency decreases and the power gain of the link noticeably increases [3].

The main target of our work is to determine an optimal geometry of the transmitting inductor to maximize the power transfer efficiency for a given receiving inductor and a given set of biological tissues. To achieve this goal, a physical model to describe the electrical properties of the printed inductors is required. In this paper we propose a lumped RLC model of printed spiral inductors (Fig. 1). Several models have been presented in literature to determine the value of such elements [4-8]. However, most of these models describe inductors integrated on silicon, thus limiting the applicability to printed inductors. In this paper, a model dedicated to printed spiral inductors has been realized starting from the different expressions presented in literature, most of which have been recently reviewed in [9]. The model has been implemented in MATLAB and the results returned by the simulations have been compared with the impedance measurements on different inductors.

The rest of the paper is organized as follows. Section 2 describes the model; Section 3 reports on the simulated geometries and on a comparison between the simulations and the measurements. Finally, Section 4 concludes the paper.

\section{DESCRIPTION OF THE MODEL}

The model aims to determine the values of the RLC lumped elements describing the behavior of a printed inductor (Fig. 1). The model receives as input the geometrical and physical 

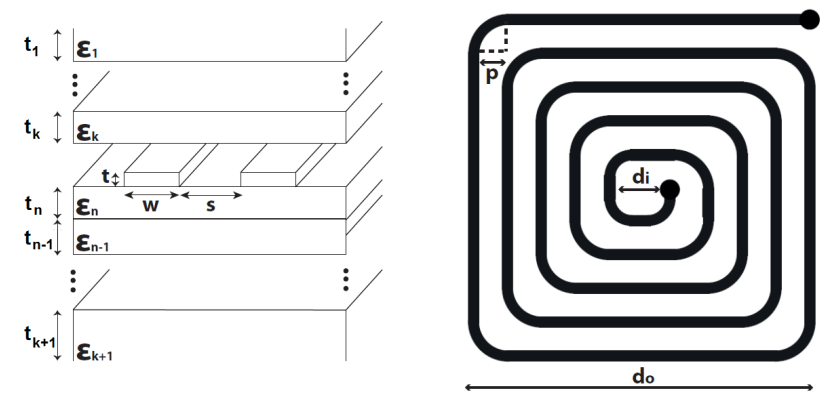

Fig. 2. Geometrical and physical parameters of a printed inductor and of the surrounding substrates.

parameters of the inductors and of the surrounding substrates (Fig. 2). In this model, only square-shaped inductors have been considered.

\section{A. Inductance $L$}

Different equations have been proposed in literature to approximate the value of the inductance $L$. We have adopted the expression proposed by [10] for squared shaped coil:

$$
L=\frac{1.27 \cdot \mu n^{2} d_{a v g}}{2}\left[\ln \left(\frac{2.07}{\varphi}\right)+0.18 \varphi+0.13 \varphi^{2}\right]
$$

where $\mu$ is the magnetic permeability of the metal traces, $n$ is the number of turns, $d_{a v g}=\left(d_{o}+d_{i}\right) / 2$, and $\varphi=\left(d_{o}-d_{i}\right) /\left(d_{o}+d_{i}\right)$. The parameter $\varphi$, called "fill factor", tends to zero if the turns are concentrated close to the external perimeter, while it tends to one if the turns fill all the area of the inductor. As underlined by [9], the error in (1) is higher than $8 \%$ for $s / w>3$ and the accuracy degrades with $\varphi \leq 0.1$ or $n \leq 2$.

\section{B. Capacitance $C_{p}$}

Parallel capacitance has an important role among the different elements of the model. Indeed, this component determines the self-resonance frequency of the inductor.

To obtain the parallel capacitance we first define the parallel capacitance per unit length $C_{a}$. According to the model presented by [7,9], the value of $C_{a}$ can be expressed as

$$
C_{a}=C_{0}+\sum_{i=1}^{n} C_{0 i}=\varepsilon_{\mathrm{eff}} C_{0}
$$

where $C_{0}$ is the capacitance per unit length between adjacent traces and $C_{0 i}$ is the capacitance between the traces and the $i^{\text {th }}$ substrate.

According to [7], the value of $C_{0}$ is defined as follows:

$$
\left\{\begin{array}{l}
C_{0}=\varepsilon_{0} \frac{K\left(k_{0}^{\prime}\right)}{K\left(k_{0}\right)} \\
k_{0}=\frac{s}{s+2 w} \\
k_{0}^{\prime}=\sqrt{1-k_{0}^{2}}
\end{array}\right.
$$

where $K(\cdot)$ is the complete elliptic integral of the first kind and $\varepsilon_{0}$ is the electrical permittivity of the vacuum $\left(\varepsilon_{0} \simeq 8.854 \times 10^{-12} \mathrm{~F} / \mathrm{m}\right)$.

The value of $\varepsilon_{\text {eff }}$ is calculated as

$$
\begin{aligned}
\varepsilon_{\mathrm{eff}}=1 & +\frac{1}{2} \sum_{i=2}^{k}\left(\varepsilon_{r_{i}}-\varepsilon_{r_{i-1}}\right) \frac{K\left(k_{0}\right) K\left(k_{i}^{\prime}\right)}{K\left(k_{0}^{\prime}\right) K\left(k_{i}\right)}+ \\
& +\frac{1}{2} \sum_{i=k+2}^{n}\left(\varepsilon_{r_{i}}-\varepsilon_{r_{i-1}}\right) \frac{K\left(k_{0}\right) K\left(k_{i}^{\prime}\right)}{K\left(k_{0}^{\prime}\right) K\left(k_{i}\right)}
\end{aligned}
$$

where the term $\varepsilon_{r_{i}}$ is the relative permittivity of the $i^{t h}$ substrate and the terms $k_{i}$ and $k_{i}^{\prime}$ are defined as

$$
\left\{\begin{array}{l}
k_{i}=\frac{\tanh \left(\frac{\pi s}{4 t_{i}}\right)}{\tanh \left(\frac{\pi(s+2 w)}{4 t_{i}}\right)} \\
k_{i}^{\prime}=\sqrt{1-k_{i}^{2}}
\end{array}\right.
$$

where $t_{i}$ is the thickness of the $i^{t h}$ substrate.

According to [7,9], the effect of the trace thickness on the capacitances is considered by reducing the spacing $s$ in the equations ( $3 \mathrm{~b}$ ) and (5a) of a factor $2 \Delta$, where $\Delta$ is defined as

$$
\Delta=\frac{t}{2 \pi \varepsilon_{e}}\left[1+\ln \left(\frac{8 \pi w}{t}\right)\right]
$$

being $\varepsilon_{e}$ the relative permittivity of the material between the traces.

Finally, by considering the total length of the inductor as

$$
l=4 n d_{o}-4 n^{2} w-(2 n-1)^{2} s+\left[\left(\frac{\pi}{2}-2\right)(4 n-1) p\right]
$$

the parallel capacitance $C_{p}$ can be written as

$$
C_{p}=\frac{C_{a}}{n} \cdot l
$$

Differently from [9], $C_{a}$ in equation (8) has been divided by a factor $n$ to consider the voltage drop across the turns of the inductors. This solution has been suggested by [11].

\section{Resistance $R_{s}$}

The series resistance of the model includes two constituents. The first constituent is independent of the frequency and is described by the Ohm's law. The second contribution is frequency dependent and includes the skin effect.

An estimation of the series resistance, suggested by [11], is

$$
R_{s}=\rho l\left(\frac{1}{w t}+\frac{1}{2 \delta(t+w)}\right)
$$

where $\rho$ is the resistivity of the metal traces. The term $\delta$ represents the skin depth and can be expressed as

$$
\delta=\sqrt{\frac{2 \rho}{\omega \mu}}
$$

where $\omega$ is the angular frequency and $\mu$ is the magnetic permeability of the metal traces. 
TABLE I

GeOMETRICAL PARAMETERS OF THE TEST INDUCTORS.

\begin{tabular}{|c|c|c|c|}
\hline Geometrical Parameters & TxA & TxB & TxC \\
\hline \hline$d_{o}(\mathrm{~mm})$ & 38 & 38 & 24.3 \\
\hline$d_{i}(\mathrm{~mm})$ & 6.6 & 6.6 & 1.4 \\
\hline$s(\mathrm{~mm})$ & 2.5 & 1 & 0.146 \\
\hline$w(\mathrm{~mm})$ & 1.2 & 1 & 0.254 \\
\hline$p(\mathrm{~mm})$ & 3.8 & 2 & 0 \\
\hline$n$ & 5 & 8 & 29 \\
\hline$t(\mu \mathrm{m})$ & 35 & 35 & 35 \\
\hline$\varepsilon_{1}(\mathrm{Air})$ & 1 & 1 & 1 \\
$t_{1}(\mathrm{~mm})$ & Open & Open & Open \\
\hline$\varepsilon_{2}(\mathrm{Air})$ & 1 & 1 & 1 \\
$t_{2}(\mathrm{~mm})$ & Open & Open & Open \\
\hline$\varepsilon_{3}(\mathrm{FR} 4)$ & 4.4 & 4.4 & 4.4 \\
$t_{3}(\mathrm{~mm})$ & 1.2 & 1.2 & 0.8 \\
\hline
\end{tabular}

\section{Resistance $R_{p}$}

Parallel resistance is due to the finite resistance of the insulating layer where the inductor is placed. According to [11], parallel resistance has been modeled as

$$
R_{p}=\frac{\rho_{k} t_{k}}{w l}
$$

where $\rho_{k}$ and $t_{k}$ are the resistivity and the thickness of the $k^{\text {th }}$ layer, where the inductor is placed.

\section{Simulations and Measurements}

The mathematical model described in the previous section has been implemented in MATLAB and the results have been compared with the measurements. The geometrical parameters of the test inductors are listed in Table I. For these geometries, the results obtained from simulations and measurements are listed in Table II. The impedance of the test inductors has been measured by means of a network analyzer, by obtaining the impedance values from the scattering parameters. In the simulations, the impedance $Z$ is determined by the lumped parameters presented in Fig. 1:

$$
Z=\frac{R_{s} R_{p}+j \omega L R_{p}}{\left(R_{s}+R_{p}-\omega^{2} L R_{p} C_{p}\right)+j \omega\left(L+R_{p} R_{s} C_{p}\right)}
$$

In Fig. 3 are shown the real and the imaginary part of the inductor impedance, comparing the results of the simulations with the measurements.

The self-resonance frequencies returned by the simulations are slightly different from those obtained with the measurements. This shift is due to the difference of the parallel capacitance $C_{p}$ between the model and the measurements. Indeed, the measured value of $C_{p}$ is noticeably influenced by several factors, such as the surrounding environment and the parasitic capacitances of the soldering. Thus, it is extremely difficult to model this element with high precision.

Nevertheless, the value of $C_{p}$ obtained with the model is important to obtain a rough estimate of the self-resonance frequency of the inductors. Then, if we assume to work at frequencies at least one order of magnitude lower than the selfresonance frequency, the model can be simplified by neglecting the presence of $C_{p}$. Indeed, at such low frequencies the parallel capacitance does not significantly affect the impedance $Z$ presented in (12).

As shown in Table II, at the operating frequency of $1 \mathrm{MHz}$ the maximum error on the impedance is $21.4 \%$ on the real part and $4.8 \%$ on the imaginary part for spiral TxA. For a more dense geometry, as in the case of spiral $\mathrm{TxC}$, the error of the model at $1 \mathrm{MHz}$ is reduced to $1.9 \%$ on the real part and $0.5 \%$ on the imaginary part.

\section{CONCLUSION}

In this paper a model of printed spiral inductors for remote powering of implantable biosensors has been presented. The model has been implemented in MATLAB and it has been compared with the measurements.

The modeling of $C_{p}$ is still unprecise. The low precision of the model is due to several factors, such as the surrounding environment and the parasitic capacitances of the soldering. Nevertheless, the values obtained with the simulations can be still used to have a rough estimate of the self-resonance frequency of the coil. By the knowledge of the self-resonance frequency, it is possible to define a range of operating frequencies where the effect of $C_{p}$ is negligible.

In that range, for the tested geometries the maximum error on the impedance is $21.4 \%$ on the real part and $4.8 \%$ on the imaginary part (TxA). For a more dense geometry $(\mathrm{TxC})$ the error of the model at $1 \mathrm{MHz}$ is reduced to $1.9 \%$ on the real part and $0.5 \%$ on the imaginary part.

Future work will focus on a better modeling of the series resistance $R_{s}$, in order to obtain a better approximation of the real part of $Z$ and to extend the validity of the model at higher frequencies.

\section{ACKNOWLEDGMENT}

J.O. thanks U. Jow from Georgia Institute of Technology for his kind helpfulness and the precious references. The research work presented in this paper was funded by the iIronIC project and by the NanoSys project. The NanoSys project is within the program ERC-2009-AdG-246810, the iIronIC project was financed with a grant from the Swiss NanoTera.ch initiative and evaluated by the Swiss National Science Foundation. 
TABLE II

ELECTRICAL PARAMETERS OF THE TEST INDUCTORS.

\begin{tabular}{|c|c|c|c|c|c|c|}
\hline \multirow{2}{*}{ Electrical Parameters } & \multicolumn{2}{|c|}{ TxA } & \multicolumn{2}{c|}{ TxB } & \multicolumn{3}{c|}{ TxC } \\
\cline { 2 - 7 } & Measurement & Model & Measurement & Model & Measurement & Model \\
\hline \hline Self-resonance frequency $(\mathrm{MHz})$ & 112.2 & 160.5 & 70.4 & 75.9 & 32.6 & 32.5 \\
\hline $\mathrm{L}(\mu \mathrm{H})$ & 0.588 & 0.565 & 1.53 & 1.52 & 9.44 & 9.50 \\
\hline $\mathrm{C}_{\mathrm{p}}(\mathrm{pF})$ & 3.42 & 1.74 & 3.34 & 2.89 & 2.52 & 2.52 \\
\hline $\mathbb{R e}\{\mathrm{Z}\} @ 1 \mathrm{MHz}(\Omega)$ & 0.28 & 0.22 & 0.56 & 0.45 & 3.61 & 3.68 \\
\hline $\operatorname{Im}\{\mathrm{Z}\} @ 1 \mathrm{MHz}(\Omega)$ & 3.76 & 3.58 & 9.76 & 9.64 & 60.1 & 60.4 \\
\hline
\end{tabular}

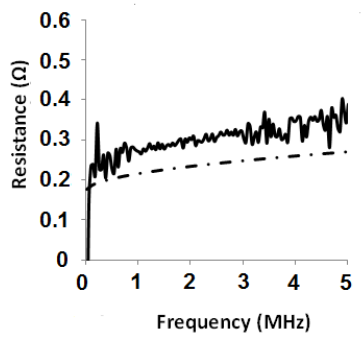

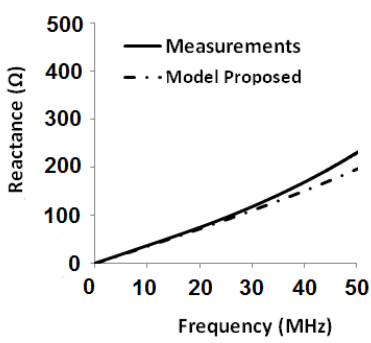

(a)

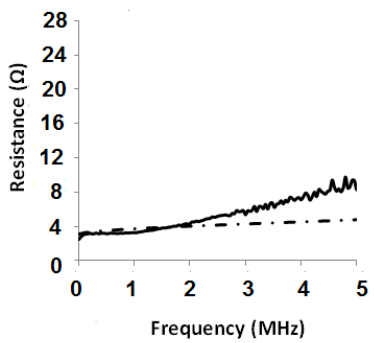

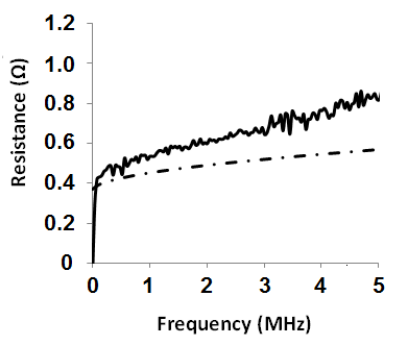

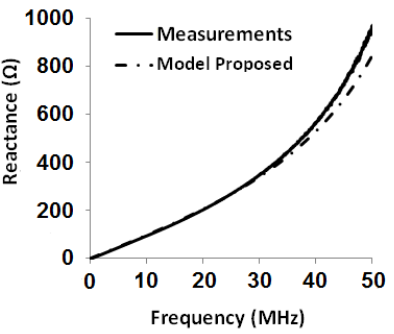

(b)

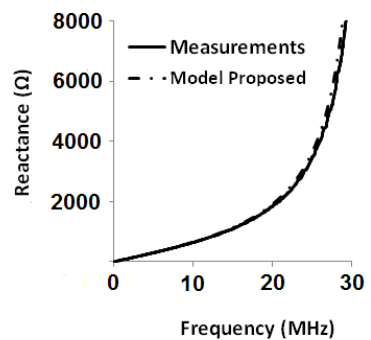

(c)

Fig. 3. Impedance of the inductors described in Table I. TxA (a), TxB (b), TxC (c)

\section{REFERENCES}

[1] B. Lenaerts and R. Puers, Omnidirectional Inductive Powering for Biomedical Implants. Springer, 2009.

[2] M. Ahmadi and G. Jullien, "A Wireless-Implantable Microsystem for Continuous Blood Glucose Monitoring," IEEE Transactions on Biomedical Circuits and Systems, vol. 3, no. 3, pp. 169 -180, June 2009.

[3] J. Olivo, S. Carrara, and G. De Micheli, "Optimal Frequencies for Inductive Powering of Fully Implantable Biosensors for Chronic and Elderly Patients," to be published in the proocedings of IEEE Sensor 2010

[4] H. Wheeler, "Formulas for Skin Effect," Proceedings of the IRE, vol. 30, no. 9, pp. 412-424, 1942.

[5] C. Zierhofer and E. Hochmair, "Geometric Approach for Coupling Enhancement of Magnetically Coupled Coils," IEEE Transaction on Biomedical Engineering, vol. 43, pp. 708-714, 1996.

[6] B. Kuhn and N. Ibrahim, "Analysis of Current Crowding Effects in Multiturn Spiral Inductors," IEEE Transactions on Microwave Theory and Techniques, vol. 49, no. 1, pp. 31-37, 2001.
[7] S. Gevorgian, H. Berg, H. Jacobsson, and T. Lewin, "Basic Parameters of Coplanar-Strip Waveguides on Multilayer Dielectric/Semiconductor Substrates, Part 1: High Permittivity Superstrates," IEEE Microwave Magazine, vol. 4, no. 2, pp. 60-70, 2003.

[8] P. Pieters, K. Vaesen, S. Brebels, S. Mahmoud, W. De Raedt, E. Beyne, and R. Mertens, "Accurate Modeling of High-Q Spiral Inductors in ThinFilm Multilayer Technology for Wireless Telecommunication Applications," IEEE Transaction on Microwave Theory and Techniques, vol. 49, no. 4, pp. 589-599, 2001.

[9] U. Jow and M. Ghovanloo, "Modeling and Optimization of Printed Spiral Coils in Air, Saline, and Muscle Tissue Environments," IEEE Transactions on Biomedical Circuits and Systems, vol. 3, no. 5, pp. 339-347, 2009.

[10] S. Mohan, M. del Mar Hershenson, and S. Boyd, "Simple Accurate Expressions for Planar Spiral Inductance," IEEE Journal of Solid-State Circuits, vol. 34, no. 10, pp. 1419-1424, 1999.

[11] C. Neagu, H. Jansen, A. Smith, J. Gardeniers, and M. Elwenspoek, "Characterization of a planar microcoil for implantable microsystems," Sensors and Actuators A: Physical, vol. 62, no. 1-3, pp. 599 - 611, 1997. 